\title{
Disorganisation, thought disorder and socio-cognitive functioning in schizophrenia spectrum disorders
}

\author{
Paulo de Sousa, William Sellwood, Martin Griffiths and Richard P. Bentall
}

\section{Background}

Poor social cognition is prevalent in schizophrenia spectrum disorders. Some authors argue that these effects are symptomspecific and that socio-cognitive difficulties (e.g. theory of mind) are strongly associated with thought disorder and symptoms of disorganisation.

\section{Aims \\ The current review tests the strength of this association.}

\section{Method}

We meta-analysed studies published between 1980 and 2016 that tested the association between social cognition and these symptoms in schizophrenia spectrum disorders.

\section{Results}

Our search (PsycINFO, MEDLINE and Web of Science) identified 123 studies ( $N=9107$ ). Overall effect size as $r=-0.313$, indicating a moderate association between symptoms and social cognition. Subanalyses yielded a moderate association between symptoms and theory of mind ( $r=-0.349)$ and emotion recognition $(r=-0.334)$, but smaller effect sizes for social perception ( $r=-0.188)$, emotion regulation $(r=-0.169)$ and attributional biases $(r=-0.143)$

\section{Conclusions}

The association is interpreted within models of communication that highlight the importance of mentalisation and processing of partner-specific cues in conversational alignment and grounding.

\section{Declaration of interests}

None.

\section{Keywords}

schizophrenia; social cognition; theory of mind; emotion recognition; emotion processing; social perception; thought disorder; disorganisation; psychosis

\section{Copyright and usage}

(c) The Royal College of Psychiatrists 2018.
'No matter how one may try, one cannot not communicate' (Watzlawick et al, 1967: pp. $48^{1}$ )

Researchers in the field of psychosis have long been interested in the role of social cognition in psychotic experiences. ${ }^{2,3}$ Consequently, there is now a wealth of meta-analytical evidence showing that deficits in theory of mind (ToM; the ability to infer mental states in others), social perception and emotion recognition are highly prevalent in individuals with schizophrenia spectrum diagnoses. ${ }^{4,5}$ Some researchers have suggested that impairments in social cognition play a specific role in disorganised symptoms in schizophrenia spectrum disorders, especially thought disorder. ${ }^{3,6}$ Here, we report a statistical synthesis of the evidence on the association between domains of social cognition and thought disorder and other symptoms of disorganisation in participants diagnosed with schizophrenia spectrum disorders.

\section{Socio-cognitive domains}

A National Institute of Mental Health workshop defined social cognition as a set of '(the) mental operations that underlie social interactions, including perceiving, interpreting, and generating responses to the intentions, dispositions, and behaviors of others' (Green et al 2008: pp. $1211^{7}$ ). Hence, social cognition is a multifaceted construct, referring to a broad range of higher-level inferential, attributional and regulatory processes, as well as lower-level social cue perception and processing. The consensus is that these processes comprise four core domains, namely: ToM and mental state attribution, social perception, attributional style or biases, and emotion processing. ${ }^{8}$ Some have distinguished a fifth domain, referred to as emotion recognition. This encompasses lower-level emotional cue perception and identification (see supplementary table a available at https://doi.org/10.1192/bjp.2018.160 for definition of domains and examples of tasks).

\section{ToM and mental state attribution}

ToM (or mental state attribution) refers to the ability of the individual to infer intentions, dispositions and beliefs in others from their speech, actions and/or non-verbal behaviour., ${ }^{3,9}$ Relevant assessment tasks may involve reading short passages describing social interactions, where intentions of the characters are inferred from hints or indirect speech acts (e.g. hinting task). ${ }^{2}$ Alternatively, participants may be asked to sequence picture-card stories that require the correct inference of false beliefs to understand the story plot (e.g. picture sequencing task). ${ }^{10}$

\section{Social perception}

Social perception refers to the ability to decode and interpret social cues (verbal and non-verbal) in an interpersonal situation. This involves both the correct interpretation of cues in a social context and the processing of social knowledge (i.e. the ability to utilise roles, rules and goals in a social situation and the knowledge of how they affect other peoples' behaviours). In some tasks, participants are presented with social situations followed by multiplechoice questions that test their ability to interpret cues about social roles and rules (e.g. Interpersonal Perception Task). ${ }^{11}$ Alternatively, tasks may involve the presentation of short audio and video clips that test the accurate interpretation of body postures, gestures, facial expressions or voice cues (e.g. Profile of Nonverbal Sensitivity Test). ${ }^{12}$

\section{Emotion recognition}

Emotion recognition refers to the ability to identify human emotion from a range of stimuli and cues, such as facial expressions or tone of voice. Emotion recognition tasks may involve the ability to correctly identify different emotional states from video clips of an actor performing facial, vocal-tonal and upper-body movement 
cues (e.g. Bell-Lysaker Emotion Recognition Task) ${ }^{13}$ or the identification of different emotional states from the tone of voice of audiotaped speakers reading out loud sentences of neutral content (e.g. Voice Emotion Identification Test). ${ }^{14}$

\section{Attributional bias/style}

Attributional bias refers to quick causal inferences that individuals make about positive and negative social events. These inferences (or attributions) are typically classified as external (i.e. the cause is attributed to others) or internal (i.e. cause is attributed to self). Sometimes, external attributions may be classified as personal (i.e. cause is the actions of another person) or situational (i.e. cause is attributed to situational factors). Tasks involve asking the participants to imagine themselves in a positive or negative social situation and to report the most likely causal explanation for an event. Example measures include the Attributional Style Questionnaire ${ }^{15}$ and the Internal, Personal, and Situational Attributions Questionnaire. ${ }^{16}$

\section{Emotion processing and regulation}

Emotion processing refers to skills that range from the perception of emotion to the understanding and management (regulation) of emotions. Although, some of these skills overlap with the competencies involved in emotion recognition, the construct is broader and encompasses affective regulatory strategies. The assessment of emotional processing can involve questionnaire measures (e.g. Emotion Regulation Questionnaire) ${ }^{17}$ or tasks where the participant is asked to rate brief vignettes that tap into the management, regulation or facilitation of emotions (e.g. Mayer-Salovey-Caruso Emotional Intelligence Test). ${ }^{18}$

\section{Thought disorder and cognitive disorganisation}

Thought disorder refers to range of thinking, linguistic and communication atypicalities that render the speech and communication of some individuals difficult to follow and apparently unintelligible. ${ }^{19}$ These symptoms are a relatively enduring feature in patients with psychosis ${ }^{20}$ and have been associated with poorer quality of life, ${ }^{21}$ higher rates of readmissions ${ }^{22}$ and poorer occupational and social functioning. ${ }^{23,24}$ Perhaps more importantly, thought disorder in patients with psychosis has been associated with poor therapeutic alliance, ${ }^{25}$ a core process in cognitive behavioural therapy for psychosis. ${ }^{26}$ Despite a considerable amount of research in the field, the processes and mechanisms involved in thought disorder are still unclear. ${ }^{27,28}$ However, such knowledge may be important for the development of effective psychological treatments for thought disorder.

Some authors have argued that no single mechanism will ever be able to explain the full range of symptoms of thought disorder because it is highly heterogeneous cluster of experiences and behaviours. ${ }^{27}$ Although, there is no final word regarding the number of factors involved in thought disorder, ${ }^{29}$ it is clear that a distinction can be made between an impoverished speech factor, which includes symptoms such as alogia (or poverty of speech), and a disorganisation factor, which includes symptoms such as derailment, tangentiality or incoherence. ${ }^{30}$ This dichotomy has also been referred to as negative and positive thought disorder. Thought disorder assessment scales, such as the Scale for the Assessment of Thought, Language and Communication Disorders (TLC) ${ }^{31}$ or the Thought Language Index, ${ }^{32}$ distinguish between poverty of speech and disorganisation items, and such differentiation has been further supported by factor analytical studies ${ }^{33}$ and studies on the psychological mechanisms of both positive and negative thought disorder. ${ }^{34,35}$

Many studies have used measurements obtained by general psychopathology scales (e.g. Positive and Negative Syndrome Scale
(PANSS) $^{36}$ or the Brief Psychiatric Rating Scale (BPRS) ${ }^{37}$ ) to test hypotheses about the mechanisms involved in thought disorder. These include single ratings of conceptual disorganisation or symptom factors. The single ratings are highly correlated with more extensive measures of thought disorder ${ }^{38}$ and they capture symptoms of disorganisation such as derailment, incoherence or illogicality (i.e. positive thought disorder) but not symptoms of cognitive impoverishment such as alogia or poverty of speech. The symptom factors, which are derived from factor analysis and are typically labelled in the literature as 'disorganisation' or 'cognitive' factors, seem to form an orthogonal cluster of experiences distinct from positive and negative symptoms in schizophrenia spectrum disorders. ${ }^{39}$ They are highly associated with positive thought disorder but not alogia or poverty of speech. ${ }^{40}$ A further problem is that they tend to encompass variance from PANSS items such as tension, inappropriate affect or mannerisms and posturing, experiences that would not normally fall under the category of thought disorder. $^{41}$

Because of the conceptual and methodological reasons outlined above, we felt that it was important that our analytical strategy distinguished between nuanced constructs, which code different and, at times, distinct phenomena.

\section{Social cognition, thought disorder and cognitive disorganisation}

One study has suggested that patients with thought disorder might be aware of their communication difficulties. ${ }^{42}$ However, some studies have reported some inconsistency between patient-reported and clinician-rated thought disorder, ${ }^{43,44}$ and others have reported that patients seem to be unaware that their verbalisations are idiosyncratic and difficult to follow despite being able to successfully judge the verbalisations of other patients with thought disorder as bizarre and atypical. ${ }^{45}$ This apparent inability to shift perspective, repair communication and cooperatively adjust the message to the needs (and level of knowledge) of the listener is crucial when communication goes awry, ${ }^{46}$ and has been highlighted by several authors as a crucial feature in thought disorder. For example, Frith $^{3}$ suggested that difficulties inferring the state of knowledge, intentions and beliefs of an interlocutor, together with difficulties in interpreting the interlocutor's social signals, could prevent repair when communication fails, thereby leading to speech being perceived by the interlocutor as tangential or derailed. Similarly, Hardy-Baylé et $a l^{6}$ suggested that symptoms of disorganisation in patients diagnosed with schizophrenia spectrum disorders could be explained by difficulties in representing other peoples' mental states and integrating contextual information during conversations. These hypotheses have been partially supported in a review ${ }^{47}$ and a meta-analysis ${ }^{5}$ of the literature on ToM in patients diagnosed with schizophrenia spectrum disorders, but difficulties with ToM do not occur in isolation from other kinds of deficits, ${ }^{48}$ and it is therefore likely that other domains of social cognition may also be important in thought disorder.

For example, Toomey et al found significant associations between poor social perception and symptoms of disorganisation in patients, ${ }^{49}$ and Kee et al found significant associations between disorganisation and poor emotion recognition. ${ }^{50}$ It is not difficult to offer interpretations of these findings. For example, stilted speech (pedantic speech that is excessively formal and inappropriate for the context of the conversation $)^{31}$ could be partially explained by poor social perception (speaking with excessive formality when the social context requires a more informal style). Although hypotheses such as these are speculative at this time, they highlight the value of exploring a wide range of domains of social cognition in relation to thought disorder and disorganisation. 


\section{Study aim}

The aim of this review was to quantify the strength of the association between different domains of social cognition and thought disorder, disorganisation and alogia in schizophrenia spectrum disorders.

\section{Method}

This review was carried out in adherence to the Meta-Analysis of Observational Studies in Epidemiology guidelines ${ }^{51}$ and the general principles of the Preferred Reporting Items for Systematic Reviews and Meta-Analyses statement for reporting systematic reviews and meta-analyses. ${ }^{52}$

\section{Literature search}

After initial scoping searches, three electronic databases (PsycINFO, MEDLINE and Web of Science) were searched for papers published between 1980 and 2016, using the following search terms: social cognition OR theory of mind OR theory-of-mind OR mentali \$ation OR mental state attribution OR affect ${ }^{\star}$ OR emotion* (recognition or identification or regulation or management or processing or perception) social perception OR social knowledge OR attribution $^{*}$ (bias* ${ }^{*}$ or style) AND schizophreni* OR psychos* AND formal thought disorder OR thought dis* OR thinking dis ${ }^{\star}$ OR disorgani ${ }^{\star}$ OR conceptual dis ${ }^{\star}$ OR cognitive dis $^{\star}$ OR communication dis $^{\star}$. The three searches yielded a total of 3077 records (Fig. 1).

\section{Study selection}

The inclusion criteria were: (a) the study was published in English language, (b) the paper was fully accessible, (c) the study was published in a peer-reviewed journal, (d) the sample was composed of patients diagnosed with schizophrenia spectrum disorders, (e) a clear thought disorder or disorganisation measure could be identified, ( $\mathrm{f}$ ) a socio-cognitive measure could be identified and (g) statistical data were available for extraction.

Although thought disorder is a transdiagnostic phenomenon that can be observed in different mental health conditions, ${ }^{27}$ we have opted to exclude studies with patients with other diagnoses (e.g. bipolar affective disorder) because there are significant differences across diagnoses on course, quality and temporal stability of these experiences. ${ }^{53-55}$

\section{Symptom grouping strategy}

To test the effect of different symptoms on social cognition, we organised the effect sizes into three different symptom groups: disorganisation (factor), alogia (poverty of speech) and thought disorder. The first group included effect sizes from studies where researchers calculated the association between social cognition and a symptom factor (e.g. 'disorganisation factor' or 'cognitive factor') derived from clinical symptom scale (e.g. PANSS or BPRS). These factors were likely to include variance from symptoms that, despite being statistically associated with thought disorder, do not represent what would normally be assumed to fall under the remit of the construct (e.g. tension, mannerisms and posturing). ${ }^{56}$ The second group (alogia) included effect sizes from studies where extractable data for the association between social cognition and a single item for alogia or poverty of speech was provided. These were almost always clinical symptom scales such as the SANS (Scale for the Assessment of Negative Symptoms). ${ }^{57}$ Finally, our third group (thought disorder) included data from studies where effect sizes were calculated from a thought disorder-specific scale score (e.g. TLC $^{58}$ or Bizarre Idiosyncratic

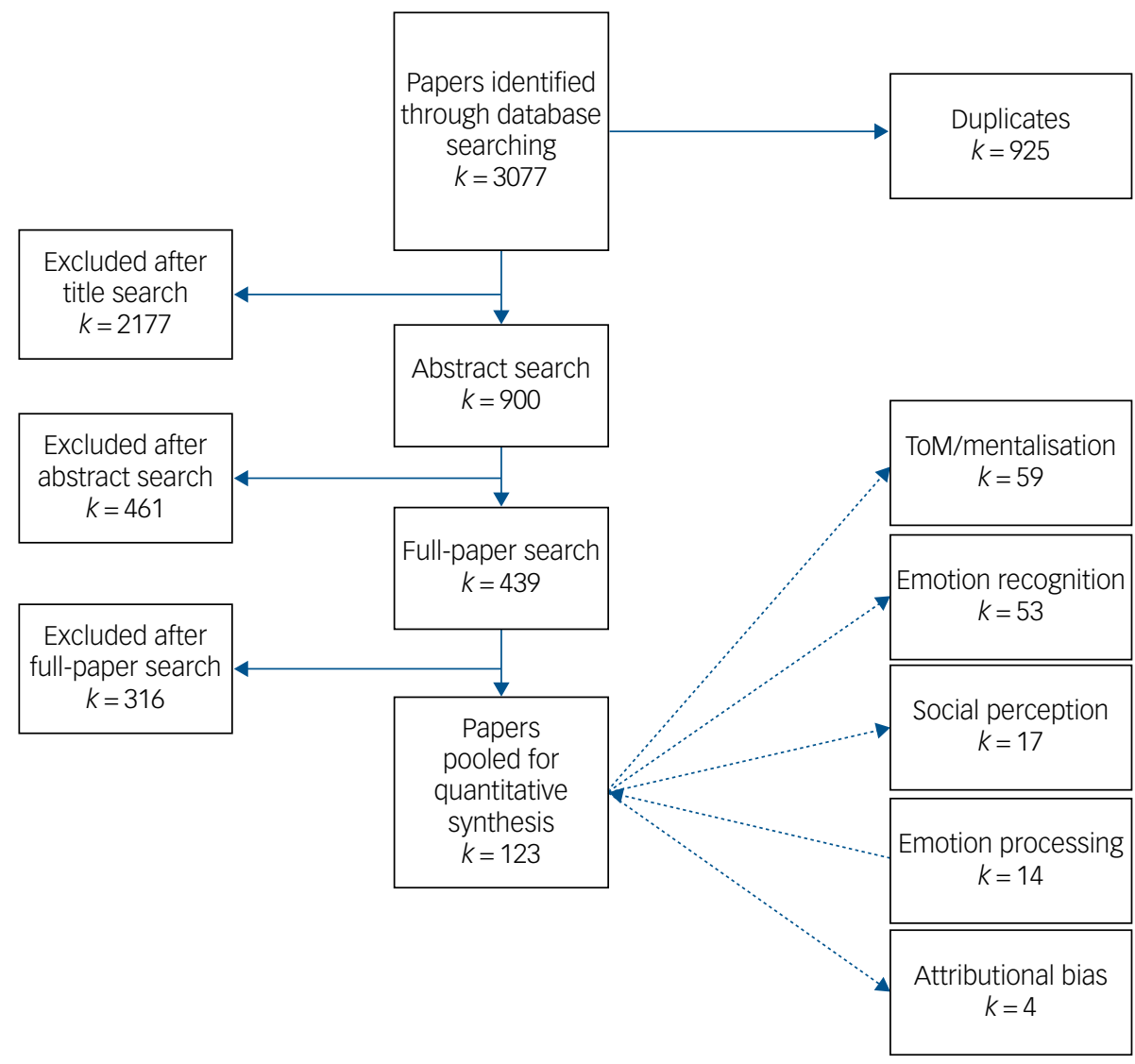

Fig. 1 Flowchart of the different stages of the systematic search. ToM, theory of mind. 
Thinking Scale ${ }^{59}$ ) or from a single item (other than alogia or poverty of speech) from a clinical rating scale (e.g. PANSS items stereotyped thinking or conceptual disorganisation $\left.{ }^{60,61}\right)$. In these cases, we opted to maintain the original designation used by the authors (see supplementary materials 6). Also included in this symptom group were effect sizes that had been estimated from clinical symptom scales that have specific thought disorder subscales (e.g. SAPS (Scale for the Assessment of Positive Symptoms) ${ }^{62}$ ). The analyses of this group included an effect size for the group as whole and then a second estimate for studies that have used only thought disorder-specific measures (without the scores from single-item clinical rating scales). The reason for this is to understand the strength of the estimate when thought disorder is measured with robust (multi-item) and purposely designed measures.

\section{Statistical analyses}

Statistical analyses were carried out with CMA (Comprehensive Meta-Analysis). Overall effect size was estimated by Pearson's correlation coefficient $(r)$ and random effects analysis, given the likelihood that our analysis would carry a substantial amount of variation across studies. In studies with multiple socio-cognitive scores within the same domain, effect size was computed from the average across tasks so that overall effect size could be computed from a single estimate by study.

Heterogeneity was measured with $\tau^{2}, Q$ and $I^{2}$, and sensitivity analysis was carried out with group comparisons and meta-regression. Publication bias was tested by the visual inspection of the funnel plot, Begg and Mazumdar's rank order correlation, Egger's regression intercept and Duval and Tweedie's 'trim and fill' procedure.

\section{Results}

\section{Study and sample characteristics}

Our search identified 123 studies with extractable data. The demographic and clinical characteristics of the studies can be found in

\begin{tabular}{|c|c|c|}
\hline \multicolumn{2}{|c|}{ Study characteristics } & \multirow{2}{*}{$\begin{array}{l}k=123 \\
114(92.68 \%) \\
9(7.32 \%)\end{array}$} \\
\hline Design & $\begin{array}{l}\text { Cross-sectional (\%) } \\
\text { Longitudinal (\%) }\end{array}$ & \\
\hline Sample size & Total & 9107 \\
\hline Gender & $\begin{array}{l}\text { Male (\%) } \\
\text { Female (\%) }\end{array}$ & $\begin{array}{l}6338(69.59 \%) \\
2573(28.25 \%)\end{array}$ \\
\hline Age & Mean (s.d.) & $36.61(6.27)$ \\
\hline Status & $\begin{array}{l}\text { Out-patient (\%) } \\
\text { In-patient (\%) } \\
\text { Mixed (\%) }\end{array}$ & $\begin{array}{l}56(45.16 \%) \\
31(25.00 \%) \\
37(29.84 \%)\end{array}$ \\
\hline $\begin{array}{l}\text { Diagnostic } \\
\text { label }\end{array}$ & $\begin{array}{l}\text { Schizophrenia (\%) } \\
\text { Spectrum (\%) }\end{array}$ & $\begin{array}{l}63(51.22 \%) \\
60(48.78 \%)\end{array}$ \\
\hline $\begin{array}{r}\text { Diagnostic } \\
\text { criteria }\end{array}$ & DSM-III-R or above (\%) & 118 (95.93\%) \\
\hline Socio-cognitive & Theory of mind (\%) & 59 (40.14\%) \\
\hline domain & Social perception (\%) & $17(11.56 \%)$ \\
\hline & Emotion recognition (\%) & $53(36.05 \%)$ \\
\hline & Attributional biases (\%) & 4 (2.72\%) \\
\hline & Emotion processing (\%) & $14(9.52 \%)$ \\
\hline Symptom & Disorganisation factor (\%) & 76 (53.15\%) \\
\hline & Alogia (\%) & $26(18.18 \%)$ \\
\hline & Thought disorder (\%) & $23(16.08 \%)$ \\
\hline & Other (\%) & $18(12.59 \%)$ \\
\hline Scale & $\begin{array}{l}\text { Positive and Negative Syndrome Scale/ } \\
\text { Scale for the Assessment of } \\
\text { Negative Symptoms/ Scale for the } \\
\text { Assessment of Positive Symptoms/ } \\
\text { Brief Psychiatric Rating Scale }\end{array}$ & $106(86.18 \%)$ \\
\hline & Other (\%) & $17(13.82 \%)$ \\
\hline
\end{tabular}

Table 1 and the methodological characteristics can be found in supplementary table $\mathrm{d}$.

\section{Overall effect size}

The pooled effect size for all the studies combined was $r=-0.313$ ( $k=123 ; 95 \%$ CI -0.346 to $-0.279 ; z=-17.226 ; P<0.001)$, which indicates a negative correlation of moderate strength. Not surprisingly, there was a significant amount of heterogeneity $(Q[122]=$ 306.702; $P<0.001 ; I^{2}=60.222 ; \tau^{2}=0.022$; s.e. $=0.006$; variance $=$ $0.000 ; \tau=0.147$ ), likely because of both the clinical and methodological diversity across studies (Fig. 2).

\section{Covariates}

To test the stability of effect sizes across time, we ran a meta-regression with year of publication as the predicting variable and individual effect size as the outcome variable. Overall, year of publication was found to be a significant predictor of the relationship between symptoms and socio-cognitive performance $(\beta=0.010$; s.e. $=$ 0.003 ; 95\% CI $0.004-0.016 ; z=3.34 ; P=0.0008)$, suggesting that effect sizes increased over time.

To test if the association between symptoms and social cognition was specific to phase of illness (i.e. state-dependent), we compared the strength of the effect size across different patient groups. The analysis of studies that have tested in-patients yielded a correlation of $-0.359(k=31 ; 95 \% \mathrm{CI}-0.419$ to $-0.297 ; z=-10.514 ; P<$ $0.001)$, with a significant level of heterogeneity $(Q[30]=44.344 ; P=$ $0.044 ; I^{2}=32.347 ; \tau^{2}=0.012 ;$ s.e. $=0.010 ;$ variance $=0.000 ; \tau=$ $0.109)$. The analysis for studies that tested out-patients yielded a smaller but nevertheless significant correlation of $-0.260 \quad(k=55$; $95 \%$ CI -0.307 to $-0.213 ; z=-10.350 ; P<0.001)$, with a significant level of heterogeneity $\left(Q[54]=120.950 ; P<0.001 ; I^{2}=55.354 ; \tau^{2}=\right.$ 0.017 ; s.e. $=0.007$; variance $=0.000 ; \tau=0.132$ ). Finally, the analysis of studies that have tested mixed samples yielded a correlation of $-0.353(k=37 ; 95 \%$ CI -0.414 to $-0.289 ; z=-10.121 ; P<$ $0.001)$, with a significant level of heterogeneity $(Q[36]=122.079$; $P<0.001 ; I^{2}=70.511 ; \tau^{2}=0.028$; s.e. $=0.014$; variance $=0.000 ; \tau=$ $0.168)$. Comparison between effect sizes revealed that differences were statistically significant $(Q[2]=8.563 ; P=0.014)$, with the effect sizes for studies with both in-patients and mixed samples being significantly higher than effect sizes for studies with out-patients.

Finally, we ran a meta-regression to test the effect of patient's age on the size of the effect size between socio-cognitive performance and thought disorder. Overall, age was not found to be a significant predictor of the effect size $(\beta=0.005$; s.e. $=0.003$; $95 \%$ CI -0.001 to $0.011 ; z=1.80 ; P=0.072$ ).

\section{Subgroup analyses by symptom}

To calculate the effect size for different symptom groups, we ran a subgroup analysis with a mixed-effects model. The analysis of studies that used disorganisation or cognitive factors derived from scales such as the PANSS and the BPRS yielded a correlation of $-0.323(k=76$; $95 \%$ CI -0.362 to $-0.282 ; z=-14.638 ; P<0.001)$, again with a significant level of heterogeneity $\left(Q[75]=205.002 ; P<0.001 ; I^{2}=63.415\right.$; $\tau^{2}=0.021$; s.e. $=0.008$; variance $=0.000 ; \tau=0.143$ ).

A subsample of studies considered alogia (or poverty of speech). For these studies, the calculation yielded a significant correlation of -0.300 ( $k=26 ; 95 \%$ CI -0.395 to $-0.198 ; z=-5.584 ; P<0.001)$, again with a significant level of heterogeneity $(Q[25]=72.995 ; P<$ $0.001 ; I^{2}=65.751 ; \tau^{2}=0.048$; s.e. $=0.023$; variance $=0.001 ; \tau=0.219$ ).

Studies that calculated the effect sizes for thought disorder (including single items such as stereotyped thinking, difficulties with abstract thinking or incoherence of speech) yielded a correlation of $-0.292(k=33 ; 95 \% \mathrm{CI}-0.350$ to $-0.232 ; z=-9.115 ; P<$ 


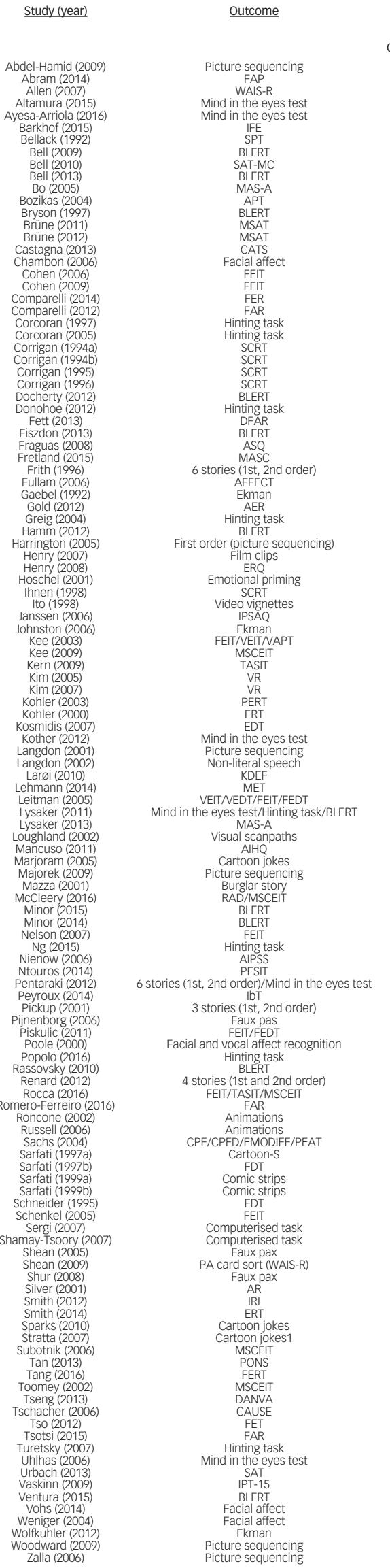

Statistics for each study

Correlation

-0.377
0.010
-0.190

-0.401
-0.063

-0.383
-0.420
-0.186

-0.070
-0.040
-0.070

-0.410
-0.700

-0.029
-0.490
-0.394

-0.033
0.050
-0.0350

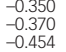

-0.299
-0.249
-0.325

-0.030
-0.450
-0.050

-.0440
-0.150
-0.150

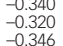

-.0210
-0.130
-0.132
-0.225

-0.212
-0.270
-0.256

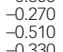

-0.330
-0.330
-0.350

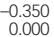

-.0 .900
-.140
-0.390
-1.040

-.5510
0.067

-.380
-0.380

-0.010
-0.300
-0.322

-0.460
-0.547

-0.066
-0.490
-0.090

-0.176
-0.480
-0.980

-0.300
-0.180
-0.520

-0.527
-0.525
-0.1850

0.240
-0.340
-0.030

-0.160
-0.120
-0.220

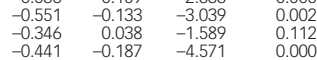

-0.220
-0.282
-0.250

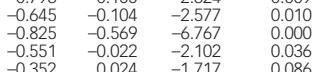

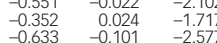

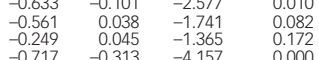

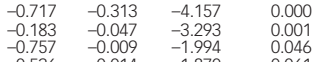

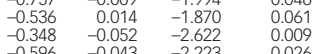

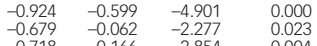

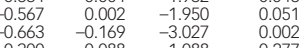

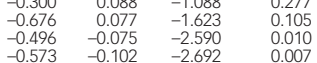

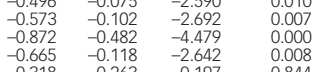

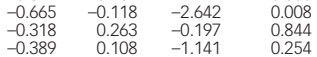

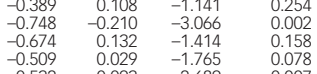

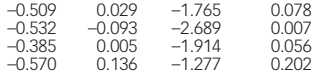

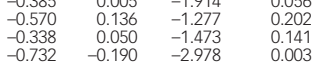

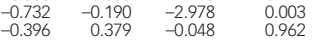

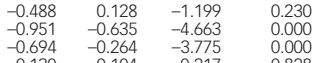

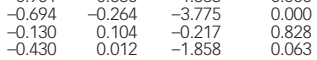

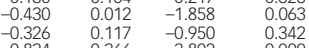

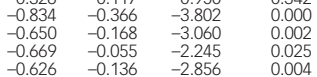

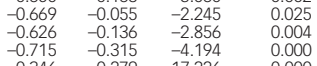

Correlation and $95 \% \mathrm{Cl}$

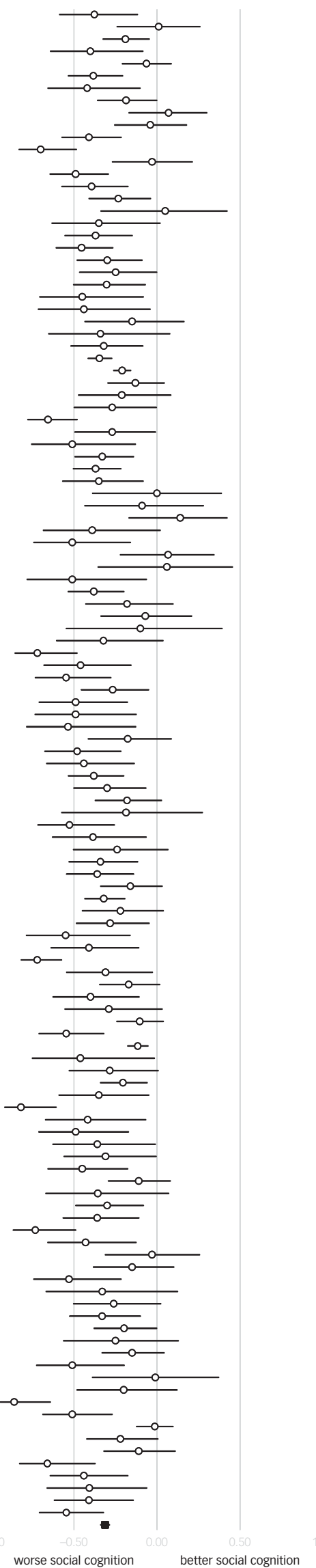

Fig. 2 Forest plot. See supplementary material 2 for full references. Picture sequencing, Picture sequencing task; FAP, Facial Affect Perception Task; WAIS-R, Picture Arrangement subtest and/or Picture Completion subtest; Mind in the eyes test, Reading the mind in the eyes test; IFE, The 
Identification of Facial Emotions Task; SPT, Social Perception Test; SAT-MC, Social Attribution Test - Multiple Choice; MAS-A, Metacognitive Assessment Scale-Abbreviated; APT, Affective Prosody Test; BLERT, Bell-Lysaker Emotion Recognition Task; MSAT, Mental State Attribution Task; CATS, Comprehensive Affect Testing System; Facial affect, Facial affect recognition task; FEIT, Facial Emotion Identification Task; FER, Facial Emotion Recognition Task; FAR, Facial Affect Recognition; Hinting task, Hinting task (ToM); SCRT, Social Cue Recognition Test; DFAR, The Degraded Facial Affect Recognition Task; ASQ, Attributional Style Questionnaire; MASC, Movie for the Assessment of Social Cognition; 6 stories, ToM Stories Task (1st and 2nd order); AFFECT, Animated Full Facial Comprehension Test; Ekman, Ekman stimuli/test; AER, Auditory Emotion Recognition Task; First order (picture sequencing), Picture sequencing task; Film clips, Emotion Elicitation using Video Clips; ERQ, Emotion Regulation Questionnaire; Emotional Priming, Emotional Priming Task; Video vignettes, Role Play Test; IPSAQ, Internal, Personal, Situational Attributions Questionnaire; VEIT, Voice Emotion Identification Test; VAPT, Videotape Affect; MSCEIT, Mayer-Salovey-Caruso Emotional Intelligence Test; TASIT, The Awareness of Social Inference Test; VR, Virtual Reality Social Perception Tool; PERT, Penn Emotion Recognition Test; ERT, Emotion Recognition Task; EDT, Emotion Discrimination Test; Non-literal speech, Picture sequencing tasks/Story Comprehension Task; KDEF, Karolinska Directed Emotional Faces; VEDT, Voice Emotion Discrimination Test; MET, Multifaceted Empathy Test; FEDT, Face Emotion Discrimination Test; Visual Scanpaths, Visual Scanpaths; AIHQ, Ambiguous Intentions Hostility Questionnaire; Cartoon jokes, Cartoon Test; Burglar story, ToM story task; RAD, Relationships Across Domains Test; AIPSS, Assessment of Interpersonal Problem-Solving Skills; PESIT, Perception of Social Inference Test; IbT, Intentionality bias Test; 3 stories, ToM Stories Task (1st and 2nd order); Faux pas, Faux Pas Task; Facial and vocal affect recognition, Facial and Vocal Affect Recognition; 4 stories, ToM Stories Task (1st and 2nd order); Animations, Animations Task; CPF, Computerised Penn Facial Memory Test; CPFD, Computerised Penn Facial Test Delayed; EMODIFF, Emotion Differentiation Test; PEAT, Penn's Emotion Acuity Test; Cartoon-S, Sarfati ToM Cartoon Stories Test; FDT, Facial Discrimination Task; Comic strips, ToM Cartoon Jokes Task; Computerised task, Computerised socio-cognitive tasks; PA card sort (WAIS-R), Picture Arrangement subtest and/or Picture Completion subtest; AR, Affective Responsiveness Task; IRI, Interpersonal Reactivity Index; PONS, Profile of Nonverbal Sensitivity Test; DANVA, Diagnostic Analysis of Nonverbal Accuracy; CAUSE, Perception of causality paradigm; IPT-15, Interpersonal Perception Task.

$0.001)$, also with a significant level of statistical heterogeneity $\left(Q[32]=47.530 ; \quad P=0.038 ; \quad I^{2}=32.675 ; \quad \tau^{2}=0.011 ; \quad\right.$ s.e. $=0.009 ;$ variance $=0.000 ; \tau=0.105)$.

To compare the effect sizes for the different symptom groups (i.e. disorganisation factor, alogia and thought disorder), we ran a mixed-effect analysis that revealed that differences between groups were not statistically significant $(Q[2]=0.758 ; P=0.684)$.

Finally, we calculated the effect sizes just for studies that had used thought disorder-specific measures (e.g. TLC). These studies yielded a correlation of -0.351 ( $k=9$; $95 \%$ CI -0.479 to -0.208 ; $z=-4.623 ; P<0.001)$, this analysis revealed a non-significant level of statistical heterogeneity $\left(Q[8]=21.924 ; P=0.005 ; I^{2}=\right.$ $63.511 ; \tau^{2}=0.033$; s.e. $=0.028$; variance $=0.001 ; \tau=0.183$ ).

TOM

The pooled effect size for the association between ToM and all symptoms combined was of moderate strength $(-0.349 ; k=59$; $95 \%$ CI -0.396 to $-0.301 ; z=-13.269 ; P<0.001)$. This association revealed a considerable amount of statistical heterogeneity $(Q[58]=$ 174.594; $P<0.001 ; I^{2}=66.780 ; \tau^{2}=0.025 ;$ s.e. $=0.010$; variance $=$ $0.000 ; \tau=0.158)$. We also analysed the data across symptom groups (see supplementary materials 5). Effect sizes for disorganisation, thought disorder and alogia were all significant and of moderate strength, with no significant difference across symptom group. The analysis for studies that used thought disorder-specific measures revealed a larger effect size with a non-significant level of heterogeneity (see supplementary materials 5 ).

\section{Social perception}

The pooled effect size for the association between social perception and symptoms was weaker $(-0.188 ; k=17 ; 95 \%$ CI -0.256 to $-0.117 ; z=-5.158 ; P<0.001)$. However, the analysis carried a non-significant amount of heterogeneity $(Q[16]=18.219 ; P=$ $0.311 ; I^{2}=12.178 ; \quad \tau^{2}=0.003 ;$ s.e. $=0.008 ;$ variance $=0.000 ; \tau=$ $0.052)$. The analyses across symptom groups revealed a significant association between social perception and thought disorder $(r=$ -0.259 ), a marginally significant and weak association with alogia and a non-significant effect size for the association between social perception and disorganisation (see supplementary materials 5).

\section{Emotion recognition}

The relationship between emotion recognition and symptoms was of moderate strength $(-0.334 ; k=53 ; 95 \%$ CI -0.380 to -0.286 ; $z=-12.842 ; P<0.001)$. Again, this analysis revealed that there was a significant amount of statistical heterogeneity across studies $\left(Q[52]=112.138 ; \quad P<0.001 ; \quad I^{2}=53.629 ; \quad \tau^{2}=0.018 ; \quad\right.$ s.e. $=0.008$; variance $=0.000 ; \tau=0.132$ ). The analyses by symptom group revealed significant and sizable effect sizes for the individual association between emotion recognition and disorganisation, thought disorder and alogia, especially with the latter $(r=-0.397)$, although differences across the three effect sizes were not significant (see supplementary materials 5).

\section{Attributional biases}

Only a small number of studies looked at attributional biases and the pooled effect size was non-significant $(-0.143 ; k=4 ; 95 \%$ CI -0.347 to $0.073 ; z=-1.298 ; P=0.194)$. Not surprisingly, this analysis revealed a very low amount of heterogeneity $(Q[3]=5.890$; $P=0.117 ; I^{2}=49.067 ; \tau^{2}=0.024 ;$ s.e. $=0.040 ;$ variance $=0.002 ; \tau=$ $0.154)$. The analyses by symptom group revealed a significant association only between attributional biases and disorganisation, but there were no significant associations for thought disorder or alogia (see supplementary materials 5).

\section{Emotion processing and regulation}

The analysis of the strength of association between emotion processing and regulation and symptoms was significant but weak $(-0.169 ; k=14 ; 95 \%$ CI -0.243 to $-0.092 ; z=-4.287 ; P<0.001)$, with a non-significant level of heterogeneity $(Q[13]=14.532 ; P=$ $0.337 ; I^{2}=10.540 ; \tau^{2}=0.002 ;$ s.e. $=0.009 ;$ variance $=0.000 ; \tau=$ 0.048 ). The analyses by symptom group revealed significant associations between emotion processing difficulties and both thought disorder and disorganisation, but not alogia (see supplementary materials 5).

\section{Publication bias}

Visual inspection of the scatterplot for the analysis including all of the studies (see supplementary figure 4) revealed some degree of asymmetry suggestive of publication bias. To test the data-set, we used the following tests: (a) Begg and Mazumdar's rank order correlation, (b) Egger's regression intercept and (c) Duval and Tweedie's trim and fill procedure.

Begg and Mazumdar's rank correlation ${ }^{63}$ yielded a significant Kendall's $\tau$ of -0.235 ( $z=3.854 ; P<0.001)$, suggestive of publication bias. Consistent with this, the Egger's test ${ }^{64}$ also yielded a significant intercept of -1.498 (s.e. $=0.275 ; 95 \%$ CI -2.042 
to $-0.955 ; t[121]=5.458 ; P<0.001)$, supporting the existence of bias. Finally, Duval and Tweedie's trim and fill procedure ${ }^{65}$ identified 35 potential missing studies (to the right of the mean). The recomputed point estimate, using a random-effects model, was -0.228 (95\% CI -0.265 to -0.191 ), suggesting that even after adjustment, the estimate was significant and sizable.

\section{Discussion}

The overall pooled effect size suggests a significant and moderate association between poor performance on socio-cognitive tasks and severity of disorganised symptoms in patients diagnosed with schizophrenia spectrum disorders. More importantly, subanalyses by symptom groups showed that correlations were sizable and significant for thought disorder, alogia and disorganised symptoms, with no significant differences between the three symptom groups. However, it is important to point out that we found a considerable amount of statistical heterogeneity. In part, this is not unexpected, given the methodological diversity in the assessments of both social cognition (e.g. emotion recognition tasks that tap into different sensory modalities or ToM tasks with different levels of complexity) and symptoms (some studies measured disorganisation with an assessment of general psychopathology, e.g. PANSS, and others measured thought disorder with specific scales, e.g. TLC). Moreover, there are considerable discrepancies across the conceptual frameworks that underlie the different thought disorder measures. ${ }^{66-68}$ Different measures rely on different ratings, scoring systems or methodologies to elicit speech samples (e.g. proverb interpretation, clinical interview, etc.), ${ }^{31,69}$ and have different clinical, cognitive and neuroanatomical correlates. ${ }^{59,70-73}$ Hence, caution is required when interpreting these findings.

One of the few analyses that did not reveal significant heterogeneity was the relationship between thought disorder and social cognition, especially in the case of the effect size calculated for studies that used thought disorder-specific measures. A possible explanation is that these studies used specific symptom measures instead of general psychopathology scales, which often only have limited items to measure cognitive disorganisation or thought disorder (e.g. PANSS or the SAPS) and may also include nonthought disorder-related items. Given that thought disorder is a heterogeneous construct, ${ }^{29}$ it is not surprising that heterogeneity was greater when more general psychopathology measures were used. In other words, the more robust the thought disorder measure, the stronger and clearer the overall effect.

Another finding that might speak to the issue of statistical heterogeneity is the association between year of publication and effect size. Our meta-regression suggested a linear and significant relationship between these two variables, with effect size increasing with time. It is possible that the emergence of dominant theories about the role of social cognition in schizophrenia spectrum disorders has inadvertently led to a publication bias toward 'positive' findings in the field. This explanation is consistent with the results of our Begg and Mazumdar's rank correlation and the Egger's test, which were consistent with the presence of publication bias, and with the trim and fill' procedure, which identified 35 potentially missing studies. However, recalculation of the point estimate after adjustment for missing studies revealed an effect size that was sizable and significant, so it seems unlikely that missing data would be sufficient to nullify the main findings.

Interestingly, the analysis by age of participants turned out to be non-significant, suggesting that the relationship between social cognition and thought disorder is relatively stable across different age groups. In contrast, the subgroup analyses by patient status revealed that effect sizes were significantly greater in studies that have tested in-patient samples. Although there is evidence suggesting that both socio-cognitive difficulties ${ }^{74}$ and thought disorder ${ }^{20}$ are not specifically characteristic of patients diagnosed with schizophrenia spectrum disorders (they can be found in other diagnostic groups), it is likely that both thought disorder and poor social cognition become more salient during periods of psychotic crisis, when patients are highly distressed. For example, it is a well-established finding that thought disorder worsens when patients are asked to talk about personally and emotionally salient topics, a phenomenon known as the affective reactivity of speech effect. ${ }^{75,76}$ It follows that if social cognition is important in thought disorder, then the relationship may well be more evident during an acute in-patient admission.

A second set of analyses concerned the effect sizes across the different socio-cognitive domains. As expected on the basis of sociocognitive theories of thought disorder and disorganisation, ${ }^{3,6}$ a strong association was found between poorer performance on ToM tasks and all symptom groups. We also found an equally sizable and significant association between poor emotion recognition and symptoms. This is not unexpected given that some ToM tasks (e.g. 'reading the mind in the eyes' test) are based on emotion recognition. However, it is interesting to note that most robust association was with alogia. In the case of social perception and emotion processing tasks, although effects were evident, they were much weaker, with former being particularly associated with positive forms of thought disorder as opposed to alogia. Regarding the weak associations with emotion processing, this is somehow unexpected given the well-reported finding that thought disorder worsens with negative affect. ${ }^{75}$ Finally, the moderate association between attributional biases and disorganisation should be interpreted with caution given that there were only two studies included in the analysis. We are aware of no theoretical model that predicts these patterns of association, but it is worth noting that some of these domains do not necessarily have absolute and categorical boundaries and may overlap greatly.

There are good theoretical reasons for expecting a relationship between thought disorder and poor social cognition. As mentioned earlier, Frith $^{3}$ suggested that communication difficulties in patients (i.e. thought disorder) could be partly explained by their inability to infer the state of knowledge of the listener. This is consistent with studies that have found that, when patients with thought disorder are provided with the opportunity to explain their perspective and contextualise their communications, their verbalisations no longer sound bizarre or 'disordered'. ${ }^{77}$ Hence, it seems reasonable to propose that difficulties at the level of social cognition (e.g. delayed activation of the frontotemporal-parietal areas that support mentalisation $)^{78}$ may render the patient unable to repair or readjust communication when unprompted, because of difficulties in timely detecting subtle and dynamic emotional and social cues from the interlocutor.

The establishment of conversational alignment, ${ }^{79}$ or grounding $^{80}$ in communication or dialog is dependent on the early, automatic and timely processing and monitoring of partner-specific information (e.g. verbal and non-verbal paralinguistic cues and signals). This process helps the addressee disambiguate language and the speaker adjust communication to the needs of the addressee, enabling the incremental shared understanding between interlocutors (as dialog unfolds) and leading to more effective and efficient communication over time. According to Brennan et $\mathrm{al}^{80}$ (pp. 316):

'(...) dialog can be viewed as a highly coordinated hypothesistesting activity that individuals engage in together, where one partner's presentation (their hypothesis of what their partner will understand) plays a dual role by providing the other 
person with evidence of how the previous utterance has been understood.'

A person who cannot disambiguate the question of the interviewer or cannot infer the state of knowledge of the listener is more likely to answer questions in an egocentric or tangential way, by intermingling, interweaving or blending in decontextualised concerns and worries into the context of the conversation, ${ }^{81}$ thereby making communications sound idiosyncratic or even bizarre. This account is consistent with findings from studies that have reported that patients who display thought disorder have significant difficulties disambiguating and processing linguistic and conversational context. ${ }^{82}$

One important point to acknowledge at this stage is that the ability to infer other peoples' mental and emotional states may not be independent from the ability to reflect and understand one's own mental state (i.e. self-reflection or meta-awareness). For example, one study showed that gains in self-reflection predicted improvements in social cognition and, more specifically, the patient's ability to infer the mental or emotional states of others. ${ }^{83}$ Some authors have hypothesised that patients with thought disorder have difficulties synthesising and making sense of their own cognitive experiences (resulting in 'cacophonous selves') ${ }^{84}$ and, consistent with this idea, two studies have reported that patients with disorganised symptoms are significantly impaired in both self-reflexivity and social cognition. ${ }^{85,86}$ There is also evidence that patients diagnosed with schizophrenia spectrum disorders have difficulties recalling autobiographical memories, ${ }^{87}$ which may be necessary when making sense of others through analogical reasoning. ${ }^{88,89}$ So it is plausible that difficulties with self-reflection or meta-awareness may underlie both poor mentalising and thought disorder. However, the relationship between poor self-reflection and other domains of social cognition also associated with thought disorder would be more difficult to explain.

Another possible interpretation is that symptoms of disorganisation may have a detrimental effect on both the patient's ability to reason about their own and other peoples' mental states. For example, Minor and colleagues reported that symptoms of disorganisation moderated the relationship between neurocognition and both social cognition and self-reflexivity in patients diagnosed with schizophrenia spectrum disorders. ${ }^{90,91}$ However, such interpretation does not explain why patients with thought disorder fail to see their verbalisation as bizarre and idiosyncratic, but can successfully judge the verbalisation of other patients with thought disorder as anomalous. ${ }^{45}$

One of the limitations of the present meta-analysis is that the calculated strength of the associations between domains of social cognition and symptoms did not account for symptom comorbidity. This is important because difficulties with ToM have been reported to be significantly associated with negative symptoms and persecutory delusions. ${ }^{5}$ In future studies, it will be important to establish the strength of the association between domains of social cognition and thought disorder after accounting for other psychotic experiences, especially negative symptoms, given its association with both poor mentalisation and dysfunctional mirror neuron activity. ${ }^{92}$ Moreover, it might be suggested that the strength of the effect size could just reflect general 'severity of illness' or more general cognitive difficulties. However, if this was the case, then one would expect the correlations with social perception, emotion regulation and attributional biases to be equally sizable, which they were not. Another limitation of the review is the overrepresentation of men in the study samples. Few studies have attempted to control or account for gender differences, so it is possible that some of these difficulties are, to some extent, gender-specific.

Social cognition is only one piece in the puzzle of thought disorder, and other psychological mechanisms have been shown to be involved in this cluster of experiences. For example, we have reported previously that difficulties in internal source monitoring (ability to correctly discriminate whether self-generated cognitions were verbalised or just thought $)^{93}$ coupled with negative affect are important to explain exacerbation of thought disorder during emotional challenge, ${ }^{75}$ and that poverty of speech seems to be specifically associated with impoverished inner speech (especially dialogical inner speech). ${ }^{35}$ Finally, how these mechanisms relate to important social predictors of thought disorder remains a matter of speculation. Some authors have suggested that difficulties recognising and reasoning about mental states in patients diagnosed with schizophrenia spectrum disorders could be a consequence of early experiences such as poor early attachments, childhood trauma or isolation, ${ }^{94}$ factors that have been found to be associated with thought disorder. ${ }^{38,95-97}$ For example, a recent study showed that poor ToM mediated the relationship between insecure attachment and emerging psychotic symptoms. ${ }^{98}$ In future studies, it will be important to examine the relationships between social predictors and socio-cognitive processes in thought disorder with more complex psychosocial models.

It may also be fruitful to test if existent socio-cognitive training packages have an effect on thought disorder (e.g. social cognition enhancement training). ${ }^{99}$ A published meta-analysis of socio-cognitive training in schizophrenia spectrum disorders reported significant and sizable effect sizes on both ToM and facial affect recognition and identification. ${ }^{100}$ The effect size for psychotic symptoms for this kind of intervention have been modest, but given the findings of the current meta-analysis, it would be pertinent to trial socio-cognitive packages that focus on both emotion recognition and perspective taking in communication on patients with persistent thought disorder. This is important given the known association between thought disorder and poorer quality of life, relapse and poorer occupational and social functioning.

Paulo de Sousa, PhD, Department of Clinical Psychology, University of Liverpool, UK William Sellwood, PhD, Division of Health Research, Faculty of Health and Medicine Lancaster University, UK; Martin Griffiths, BSC, Department of Clinical Psychology, University of Liverpool, UK; Richard P. Bentall, PhD, Clinical Psychology Unit Department of Psychology, University of Sheffield, UK

Correspondence: Paulo Sousa, Department of Clinical Psychology, University of Liverpool, Whelan Building, The Quadrangle, Brownlow Hill, Liverpool L69 3GB, UK. Email: sousa@liv.ac.uk

First received 17 Apr 2018, final revision 20 Jun 2018, accepted 17 Jul 2018

\section{Supplementary material}

Supplementary material is available online at https://doi.org/10.1192/bjp.2018.160

\section{References}

1 Watzlawick P, Bavelas JB, Jackson DD. Pragmatics of Human Communication: A Study of Interactional Patterns, Pathologies and Paradoxes. W. W. Norton \& Company, 1967.

2 Corcoran R, Mercer G, Frith CD. Schizophrenia, symptomatology and social inference: investigating 'theory of mind' in people with schizophrenia. Schizophr Res 1995; 17(1): 5-13.

3 Frith CD. The Cognitive Neuropsychology of Schizophrenia. Psychology Press, 1992

4 Savla GN, Vella L, Armstrong CC, Penn DL, Twamley EW. Deficits in domains of social cognition in schizophrenia: a meta-analysis of the empirical evidence. Schizophr Bull 2013; 39: 979-92.

5 Sprong M, Schothorst $\mathrm{P}$, VoS E, Hox J, van Engeland H. Theory of mind in schizophrenia: meta-analysis. Br J Psychiatry 2007; 191: 5-13.

6 Hardy-Baylé M-C, Sarfati Y, Passerieux C. The cognitive basis of disorganization symptomatology in schizophrenia and its clinical correlates. Schizophr Bull 2003; 29(3): 459-71. 
7 Green MF, Penn DL, Bentall R, Carpenter WT, Gaebel W, Gur RC, et al. Social cognition in schizophrenia: an NIMH workshop on definitions, assessment, and research opportunities. Schizophr Bull 2008; 34(6): 1211-20.

8 Murray L, Hentges F, Hill J, Karpf J, Mistry B, Kreutz M, et al. The effect of cleft lip and palate, and the timing of lip repair on mother-infant interactions and infant development. J Child Psychol Psychiatry Allied Discip 2008; 49: 115-23.

9 Baron-Cohen S, Wheelwright S, Hill J, Raste Y, Plumb I. The 'Reading the Mind in the Eyes' Test revised version: a study with normal adults, and adults with Asperger syndrome or high-functioning autism. J Child Psychol Psychiatry 2001; 42: 241-51.

10 Langdon R, Coltheart M. Mentalising, schizotypy, and schizophrenia. Cognition 1999; 71: 43-71.

11 Costanzo M, Archer D. Interpreting the expressive behavior of others: the Interpersonal Perception Task. J Nonverbal Behav 1989; 13: 225-45.

12 Rosenthal JA, DiMatteo MR, Rogers PL, Archer D. Sensitivity to Nonverbal Communication: The PONS Test. Johns Hopkins University Press, 1979.

13 Bryson G, Bell M, Lysaker P. Affect recognition in schizophrenia: a function of global impairment or a specific cognitive deficit. Psychiatry Res 1997; 71: 105-13.

14 Kerr SL, Neale JM. Emotion perception in schizophrenia: specific deficit or further evidence of generalized poor performance? J Abnorm Psychol 1993; 102: $312-8$.

15 Peterson C, Semmel A, von Baeyer C, Abramson LY, Metalsky GI, Seligman MEP. The attributional Style Questionnaire. Cognit Ther Res 1982; 6 (3): 287-99.

16 Kinderman P, Bentall RP. A new measure of causal locus: the internal, personal and situational attributions questionnaire. Pers Individ Dif 1996; 20: 261-4.

17 Gross JJ, John OP. Individual differences in two emotion regulation processes: implications for affect, relationships, and well-being. J Pers Soc Psychol 2003; 85: 348-62.

18 Mayer JD, Salovey P, Caruso DR. Mayer-Salovey-Caruso Emotional Intelligence Test. Multi-Health Systems, Inc., 1999

19 Andreasen NC. Should the term 'thought disorder' be revised? Compr Psychiatry 1982; 23(4): 291.

20 Marengo J, Harrow M. Longitudinal courses of thought disorder in schizophrenia and schizoaffective disorder. Schizophr Bull 1997; 23: 273-85.

21 Tan EJ, Thomas N, Rossell SL. Speech disturbances and quality of life in schizophrenia: differential impacts on functioning and life satisfaction. Compr Psychiatry 2014; 55: 693-8.

22 Harrow M, Marengo JT. Schizophrenic thought disorder at followup: its persistence and prognostic significance. Schizophr Bull 1986; 12: 373-93.

23 Racenstein JM, Penn D, Harrow M, Schleser R. Thought disorder and psychosocial functioning in schizophrenia: the concurrent and predictive relationships. J Nerv Ment Dis 1999; 187: 281-9.

24 Bowie CR, Gupta M, Holshausen K. Disconnected and underproductive speech in schizophrenia: unique relationships across multiple indicators of social functioning. Schizophr Res 2011; 131: 152-6.

25 Cavelti M, Homan P, Vauth R. The impact of thought disorder on therapeutic alliance and personal recovery in schizophrenia and schizoaffective disorder: an exploratory study. Psychiatry Res 2016; 239: 92-8.

26 Goldsmith LP, Lewis SW, Dunn G, Bentall RP. Psychological treatments for early psychosis can be beneficial or harmful, depending on the therapeutic alliance: an instrumental variable analysis. Psychol Med 2015; 45: 2365-73.

27 McKenna PJ, Oh TM. Schizophrenic Speech: Making Sense of Bathroots and Ponds That Fall in Doorways. Cambridge University Press, 2005.

28 Bentall RP, De Sousa P, Varese F, Wickham S, Sitko K, Haarmans M, et al. From adversity to psychosis: pathways and mechanisms from specific adversities to specific symptoms. Soc Psychiatry Psychiatr Epidemiol 2014; 49(7): 1011-22.

29 Roche E, Creed L, MacMahon D, Brennan D, Clarke M. The epidemiology and associated phenomenology of formal thought disorder: a systematic review. Schizophr Bull 2015; 41(4): 951-62.

30 Andreasen NC, Grove WM. Thought, language, and communication in schizophrenia: diagnosis and prognosis. Schizophr Bull 1986; 12(3): 348-59.

31 Andreasen NC. Scale for the assessment of thought, language, and communication (TLC). Schizophr Bull 1986; 12(3): 473-82.

32 Liddle PF, Ngan ETC, Caissie SL, Anderson CM, Bates AT, Quested DJ, et al. Thought and Language Index: an instrument for assessing thought and language in schizophrenia. Br J Psychiatry 2002; 181(4): 326-30.

33 Harvey P, Lenzenweger MF, Keefe RSE, Pogge DL, Serper MR, Mohs RC. Empirical assessment of the factorial structure of clinical symptoms in schizophrenic patients: formal thought disorder. Psychiatry Res 1992; 44(2): 141-51.

34 Docherty AR, Berenbaum H, Kerns JG. Alogia and formal thought disorder: differential patterns of verbal fluency task performance. J Psychiatr Res 2011; 45(10): 1352-7
35 de Sousa P, Sellwood W, Spray A, Fernyhough C, Bentall RP. Inner speech and clarity of self-concept in thought disorder and auditory-verbal hallucinations. J Nerv Ment Dis 2016; 204(12): 885-93.

36 Kay SR, Fiszbein A, Opler LA. The Positive And Negative Syndrome Scale (PANSS) for schizophrenia. Schizophr Bull 1987; 13(2): 261-76.

37 Overall JE, Gorham DR. The Brief Psychiatric Rating Scale. Psychol Rep 1962; 10 (3): 799-812.

38 de Sousa P, Spray A, Sellwood W, Bentall RP. 'No man is an island'. Testing the specific role of social isolation in formal thought disorder. Psychiatry Res 2015; 230(2): 304-13.

39 Grube BS, Bilder RM, Goldman RS. Meta-analysis of symptom factors in schizophrenia. Schizophr Res 1998; 31(2-3): 113-20.

40 Peralta V, Cuesta M, de Leon J. Formal thought disorder in schizophrenia: a factor analytic study. Compr Psychiatry 1992; 33(2): 105-10.

41 Liddle PF. The symptoms of chronic schizophrenia. A re-examination of the positive-negative dichotomy. Br J Psychiatry 1987; 151: 145-51.

42 McGrath J, Allman R. Awareness and unawareness of thought disorder. Aust N Z J Psychiatry 2000; 34: 35-42.

43 Barrera A, McKenna PJ, Berrios GE. Two new scales of formal thought disorder in schizophrenia. Psychiatry Res 2008; 157(1): 225-34.

44 Barrera Á, McKenna PJ, Berrios GE. Formal thought disorder, neuropsychology and insight in schizophrenia. Psychopathology 2009; 42(4): 264-9.

45 Harrow M, Lanin-Kettering I, Miller JG. Impaired perspective and thought pathology in schizophrenic and psychotic disorders. Schizophr Bull 1989; 15: 605-23.

46 Pickering MJ, Garrod S. Toward a mechanistic psychology of dialogue. Behav Brain Sci 2004; 27: 169-90; discussion 190-226.

47 Brüne M. 'Theory of mind' in schizophrenia: a review of the literature. Schizophr Bull 2005; 31: 21-42.

48 Green MF, Horan WP, Lee J. Social cognition in schizophrenia. Nat Rev Neurosci 2015; 16: 620-31.

49 Toomey R, Schuldberg D, Corrigan P, Green MF. Nonverbal social perception and symptomatology in schizophrenia. Schizophr Res 2002; 53 (1-2): 83-91.

50 Kee K, Green M, Mintz J, Brekke J. Is emotion processing a predictor of functional outcome in schizophrenia? Schizophr Bull 2003; 29(3): 487-97.

51 Stroup DF, Berlin JA, Morton SC, Olkin I, Williamson GD, Rennie D, et al. Metaanalysis of observational studies in epidemiology: a proposal for reporting. Meta-analysis Of Observational Studies in Epidemiology (MOOSE) group. JAMA 2000; 283(15): 2008-12.

52 Moher D, Liberati A, Tetzlaff J, Altman DG. Preferred reporting items for systematic reviews and meta-analyses: the PRISMA statement. BMJ 2009; 339 b2535.

53 Holzman PS, Shenton M, Solovay MR. Quality of thought disorder in differential diagnosis. Schizophr Bull 1986; 12(3): 360-71.

54 Solovay MR, Shenton M, Holzman PS. Comparative studies of thought disorders: I. Mania and schizophrenia. Arch Gen Psychiatry 1987; 44(1): 13.

55 Yalincetin B, Bora E, Binbay T, Ulas H, Akdede BB, Alptekin K. Formal thought disorder in schizophrenia and bipolar disorder: a systematic review and metaanalysis. Schizophr Res 2016; 185: 2-8.

56 Stratta P, Riccardi I, Mirabilio D, Di Tommaso S, Tomassini A, Rossi A Exploration of irony appreciation in schizophrenia: a replication study on an Italian sample. Eur Arch Psychiatry Clin Neurosci 2007; 257(6): 337-9.

57 Bell MD, Corbera S, Johannesen JK, Fiszdon JM, Wexler BE. Social cognitive impairments and negative symptoms in schizophrenia: are there subtypes with distinct functional correlates? Schizophr Bull 2013; 39(1): 186-96.

58 Sarfati Y, Hardy-Baylé MC, Besche C, Widlöcher D. Attribution of intentions to others in people with schizophrenia: a non- verbal exploration with comic strips. Schizophr Res 1997; 25: 199-209.

59 Subotnik KL, Nuechterlein KH, Green MF, Horan WP, Nienow TM, Ventura J, et al. Neurocognitive and social cognitive correlates of formal thought disorder in schizophrenia patients. Schizophr Res 2006; 85: 84-95.

60 Kim K, Kim J-J, Kim J, Park D-E, Jang HJ, Ku J, et al. Characteristics of social perception assessed in schizophrenia using virtual reality. Cyberpsychol Behav 2007; 10(2): 215-9.

61 Kim K, Kim J-J, Kim J, Ku JH, Jang HJ, Park SH, et al. Investigation of social cue perception in schizophrenia using virtual reality. Annu Rev CyberTherapy Telemed 2005; 3: 135-42.

62 Chambon V, Baudouin JY, Franck N. The role of configural information in facial emotion recognition in schizophrenia. Neuropsychologia 2006; 44(12): 2437-44.

63 Begg CB, Mazumdar M. Operating characteristics of a rank correlation test for publication bias. Biometrics 1994; 50: 1088-101. 
64 Egger M, Davey Smith G, Schneider M, Minder C. Bias in meta-analysis detected by a simple, graphical test. BMJ 1997; 315: 629.

65 Duval S, Tweedie R. Trim and fill: a simple funnel-plot-based method of testing and adjusting for publication bias in meta-analysis. Biometrics 2000; 56: 455-63.

66 Chaika E. Thought disorder or speech disorder in schizophrenia? Schizophr Bull 1982; 8(4): 587-91.

67 Harrod JB. Schizophrenia as a semiotic disorder. Schizophr Bull 1986; 12(1): 12-3.

68 Lanin-Kettering I, Harrow M. The thought behind the words: a view of schizophrenic speech and thinking disorders. Schizophr Bull 1985; 11: 1-15.

69 Marengo J, Harrow M, Lanin-Kettering I, Wilson A. Evaluating bizarre-idiosyncratic thinking: a comprehensive index of positive thought disorder. Schizophr Bull 1986; 12(3): 497-511.

70 Sponheim SR, Surerus-Johnson C, Leskela J, Dieperink ME. Proverb interpretation in schizophrenia: the significance of symptomatology and cognitive processes. Schizophr Res 2003; 65(2-3): 117-23.

71 Roche E, Segurado R, Renwick L, McClenaghan A, Sexton S, Frawley T, et al. Language disturbance and functioning in first episode psychosis. Psychiatry Res 2015; 235: 29-37.

72 Subotnik KL, Bartzokis G, Green MF, Nuechterlein KH. Neuroanatomical correlates of formal thought disorder in schizophrenia. $\operatorname{cogn}$ Neuropsychiatry 2003; 8: 81-8.

73 Docherty N. Cognitive impairments and disordered speech in schizophrenia: thought disorder, disorganization, and communication failure perspectives. J Abnorm Psychol 2005; 114(2): 269-78.

74 Mccleery A, Lee J, Fiske AP, Ghermezi L, Hayata JN, Hellemann GS, et al Longitudinal stability of social cognition in schizophrenia: a 5-year follow-up of social perception and emotion processing. Schizophr Res 2016; 176(2-3): $467-$ 72.

75 de Sousa P, Sellwood W, Spray A, Bentall RP. The affective reactivity of psychotic speech: the role of internal source monitoring in explaining increased thought disorder under emotional challenge. Schizophr Res 2016; 172: 189-94.

76 Docherty N. Affective reactivity of symptoms as a process discriminator in schizophrenia. J Nerv Ment Dis 1996; 184: 535-41.

77 Harrow M, Quinlan DM. Disordered Thinking and Schizophrenic Psychopathology. Gardner Press, 1985.

78 Pedersen A, Koelkebeck K, Brandt M, Wee M, Kueppers KA, Kugel H, et al. Theory of mind in patients with schizophrenia: is mentalizing delayed? Schizophr Res 2012; 137(1-3): 224-9.

79 Pickering MJ, Garrod S. Alignment as the basis for successful communication. Res Lang Comput 2006; 4: 203-28.

80 Brennan SE, Galati A, Kuhlen AK. Two minds, one dialog: coordinating speaking and understanding. Psychol Learn Motiv 2010; 53: 301-44.

81 Harrow M, Lanin-Kettering I, Prosen M, Miller JG. Disordered thinking in schizophrenia: intermingling and loss of set. Schizophr Bull 1983; 9: 354-67.

82 Kuperberg GR, McGuire PK, David AS. Reduced sensitivity to linguistic context in schizophrenic thought disorder: evidence from on-line monitoring for words in linguistically anomalous sentences. J Abnorm Psychol 1998; 107 423-34.

83 Dimaggio G, Lysaker PH, Carcione A, Nicolò G, Semerari A. Know yourself and you shall know the other... to a certain extent: multiple paths of influence of self-reflection on mindreading. Conscious Cogn 2008; 17(3): 778-89.
84 Lysaker PH, Lysaker JTJT. Schizophrenia and the collapse of the dialogical self: recovery, narrative and psychotherapy. Psychother Theory Res Pract Train 2001; 38: 252-61.

85 Lysaker PH, Gumley A, Brüne M, Vanheule S, Buck KD, Dimaggio G. Deficits in the ability to recognize one's own affects and those of others: associations with neurocognition, symptoms and sexual trauma among persons with schizophrenia spectrum disorders. Conscious Cogn 2011; 20(4): 1183-92.

86 Lysaker PH, Gumley A, Luedtke B, Buck KD, Ringer JM, Olesek K, et al. Socia cognition and metacognition in schizophrenia: evidence of their independence and linkage with outcomes. Acta Psychiatr Scand 2013; 127(3): 239-47.

87 Berna F, Potheegadoo J, Aouadi I, Ricarte JJ, Allé MC, Coutelle R, et al. A metaanalysis of autobiographical memory studies in schizophrenia spectrum disorder. Schizophr Bull 2016; 42(1): 56-66.

88 Saxe R, Moran JM, Scholz J, Gabrieli J. Overlapping and non-overlapping brain regions for theory of mind and self reflection in individual subjects. Soc Cogn Affect Neurosci 2006; 1(3): 229-34.

89 Corcoran R, Frith CD. Autobiographical memory and theory of mind: evidence of a relationship in schizophrenia. Psychol Med 2003; 33(5): 897-905.

90 Minor KS, Lysaker PH. Necessary, but not sufficient: links between neurocognition, social cognition, and metacognition in schizophrenia are moderated by disorganized symptoms. Schizophr Res 2014; 159(1): 198-204.

91 Minor KS, Marggraf MP, Davis BJ, Luther L, Vohs JL, Buck KD, et al. Conceptual disorganization weakens links in cognitive pathways: disentangling neurocognition, social cognition, and metacognition in schizophrenia. Schizophr Res 2015; 169(1-3): 153-8.

92 Mehta UM, Thirthalli J, Aneelraj D, Jadhav P, Gangadhar BN, Keshavan MS. Mirror neuron dysfunction in schizophrenia and its functional implications: a systematic review. Schizophr Res 2014; 160(1-3): 9-19.

93 Johnson MK, Hashtroudi S, Lindsay DS. Source monitoring. Psychol Bull 1993: 114: 3-28.

94 Dimaggio G, Popolo R, Salvatore G, Lysaker PH. Mentalizing in schizophrenia is more than just solving theory of mind tasks. Front Psychol 2013; 4: 83.

95 Read J, Argyle N. Hallucinations, delusions, and thought disorder among adult psychiatric inpatients with a history of child abuse. Psychiatr Serv 1999; 50(11): 1467-72.

96 Toth SL, Pickreign Stronach E, Rogosch FA, Caplan R, Cicchetti D. Illogical thinking and thought disorder in maltreated children. J Am Acad Child Adolesc Psychiatry 2011; 50(7): 659-68.

97 Dozier M, Lee SW. Discrepancies between self-and other-report of psychiatric symptomatology: effects of dismissing attachment strategies. Dev Psychopathol 1995; 7(01): 217-26.

98 Hart JR, Venta A, Sharp C. Attachment and thought problems in an adolescent inpatient sample: the mediational role of theory of mind. Compr Psychiatry 2017; 78: 38-47

99 Choi KH, Kwon JH. Social cognition enhancement training for schizophrenia: a preliminary randomized controlled trial. Community Ment Health J 2006; 42(2): $177-87$.

100 Kurtz MM, Richardson CL. Social cognitive training for schizophrenia: a meta-analytic investigation of controlled research. Schizophr Bull 2012 38(5): 1092-104. 